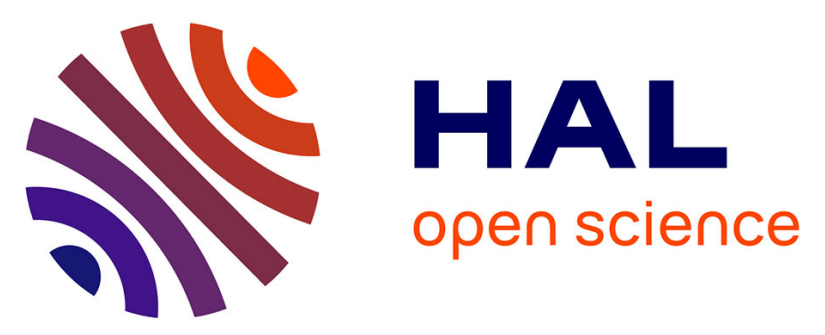

\title{
Synergy of Excitation Enhancement and the Purcell Effect for Strong Photoluminescence Enhancement in a Thin-Film Hybrid Structure based on Quantum Dots and Plasmon Nanoparticles
}

Victor Krivenkov, Pavel S Samokhvalov, Igor R Nabiev, Yury P Rakovich

\section{To cite this version:}

Victor Krivenkov, Pavel S Samokhvalov, Igor R Nabiev, Yury P Rakovich. Synergy of Excitation Enhancement and the Purcell Effect for Strong Photoluminescence Enhancement in a Thin-Film Hybrid Structure based on Quantum Dots and Plasmon Nanoparticles. Journal of Physical Chemistry Letters, 2020, 11 (19), pp.8018-8025. 10.1021/acs.jpclett.0c02296 . hal-02933842

\author{
HAL Id: hal-02933842 \\ https://hal.science/hal-02933842
}

Submitted on 8 Sep 2020

HAL is a multi-disciplinary open access archive for the deposit and dissemination of scientific research documents, whether they are published or not. The documents may come from teaching and research institutions in France or abroad, or from public or private research centers.
L'archive ouverte pluridisciplinaire HAL, est destinée au dépôt et à la diffusion de documents scientifiques de niveau recherche, publiés ou non, émanant des établissements d'enseignement et de recherche français ou étrangers, des laboratoires publics ou privés. 
Subscriber access provided by bibliothèque universitaire | Université de Reims Champagne Ardenne

\title{
Physical Insights into Light Interacting with Matter
}

\section{Synergy of Excitation Enhancement and the Purcell Effect for Strong Photoluminescence Enhancement in a Thin-Film Hybrid Structure based on Quantum Dots and Plasmon Nanoparticles.}

\author{
Victor Krivenkov, Pavel S. Samokhvalov, Igor R. Nabiev, and Yury P. Rakovich
}

J. Phys. Chem. Lett., Just Accepted Manuscript • DOI: 10.1021/acs.jpclett.0c02296 • Publication Date (Web): 04 Sep 2020

Downloaded from pubs.acs.org on September 8, 2020

\section{Just Accepted}

"Just Accepted" manuscripts have been peer-reviewed and accepted for publication. They are posted online prior to technical editing, formatting for publication and author proofing. The American Chemical Society provides "Just Accepted" as a service to the research community to expedite the dissemination of scientific material as soon as possible after acceptance. "Just Accepted" manuscripts appear in full in PDF format accompanied by an HTML abstract. "Just Accepted" manuscripts have been fully peer reviewed, but should not be considered the official version of record. They are citable by the Digital Object Identifier (DOI®). "Just Accepted" is an optional service offered to authors. Therefore, the "Just Accepted" Web site may not include all articles that will be published in the journal. After a manuscript is technically edited and formatted, it will be removed from the "Just Accepted" Web site and published as an ASAP article. Note that technical editing may introduce minor changes to the manuscript text and/or graphics which could affect content, and all legal disclaimers and ethical guidelines that apply to the journal pertain. ACS cannot be held responsible for errors or consequences arising from the use of information contained in these "Just Accepted" manuscripts. 


\section{Synergy of Excitation Enhancement and the Purcell

\author{
Effect for Strong Photoluminescence Enhancement
}

in a Thin-Film Hybrid Structure based on Quantum

\section{Dots and Plasmon Nanoparticles}

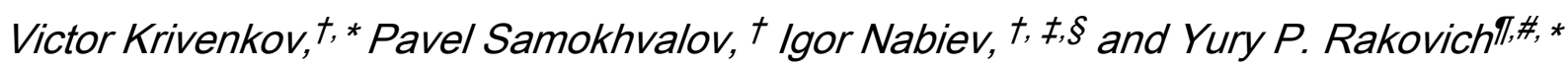

† National Research Nuclear University MEPhl (Moscow Engineering Physics Institute),

115409 Moscow, Russian Federation

‡Laboratoire de Recherche en Nanosciences, LRN-EA4682, Université de Reims

Champagne-Ardenne, 51100 Reims, France

$\S$ Sechenov First Moscow State Medical University (Sechenov University), 119146

Moscow, Russian Federation 
ๆ Centro de Física de Materiales (MPC, CSIC-UPV/EHU), Donostia International

Physics Center (DIPC), and Departamento de Física de Materiales, UPV-EHU, 20018

Donostia - San Sebastian, Spain

\# IKERBASQUE, Basque Foundation for Science, 48013 Bilbao, Spain

\title{
Corresponding Authors
}

*vkrivenkov@list.ru (VK) or yury.rakovich@ehu.eus (YR)

\author{
ABSTRACT
}

Reliable control of spontaneous radiation from quantum emitters, such as quantum dots (QDs), is an extremely important problem in quantum science, nanophotonics, and engineering. The QD photoluminescence (PL) may be enhanced near plasmon nanoparticles due to excitation field enhancement or the Purcell effect. However, both of these effects have their specific limitations. The excitation enhancement is usually accompanied by a decrease in the PL quantum yield (QY) due to the plasmon-induced energy transfer, and the Purcell effect cannot significantly enhance the PL of QDs with an initially high QY because of the obvious limitation of the QY by the value of $100 \%$. Here, we have shown that the synergistic combination of excitation enhancement 
caused by silver nanospheres and the Purcell effect caused by silver nanoplates in the same QD-in-polymer hybrid thin-film nanostructure permits simultaneous increases in the radiative and excitation rates to be obtained. This overcomes the limitations of each individual effect and yields a synergistic PL increase $(+1320 \%)$ higher than the sum of the PL enhancements determined by each effect alone $(+70 \%$ and $+360 \%)$.

\section{TOC GRAPHIC}

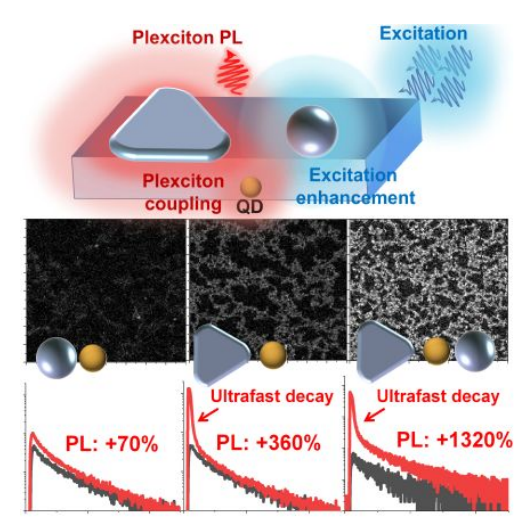

KEYWORDS Photoluminescence enhancement, Purcell effect, near-field enhancement, quantum dots, plasmonics.

During recent decades, the use of localized surface plasmons (hereinafter, plasmons) has been gaining momentum in the field of nanophotonics due to some unique nearfield effects. In particular, two very interesting features of plasmons may be used to enhance fluorophore photoluminescence (PL): local excitation field enhancement and 
the Purcell effect. ${ }^{1,2}$ The condition for the enhancement of the excitation field near plasmon nanostructures is resonant overlapping of the field frequency with the eigenfrequency of the plasmons. ${ }^{3}$ On the other hand, the requirement for accelerating the radiative recombination (the Purcell effect) is a resonant overlap of the plasmon frequency with the PL band of the emitter. ${ }^{4,5}$ While the use of optical microcavities is a common method for increasing the spontaneous emission of quantum emitters, ${ }^{6}$ the density of photonic states near plasmon nanostructures can be several orders of magnitude higher. This allows a significant increase in the excitation efficiency compared with microcavities. Moreover, the limit of the radiative decay acceleration of the emitter near plasmon nanostructures is two orders of magnitude higher than the theoretical limit for microcavities. ${ }^{7}$

The emergence of new emitting nanoscale exciton nanostructures, such as semiconductor quantum dots (QDs), raises new challenges in the development of hybrid materials based on plasmon nanostructures. One of the most important applications of QDs for advanced optoelectronic and optical information devices, ${ }^{8-10}$ as well as for chemo- and biosensors, ${ }^{11}$ is the design of fluorescent thin-film coatings using polymer matrices with integrated QDs and plasmon nanoparticles (PNPs). For these 
applications, the most critical parameters of QDs are the PL intensity (brightness) and lifetime. Both of these parameters can be improved through the interaction with plasmons. $^{12}$ In previous studies, it was experimentally shown that an enhancement of the QD PL intensity by a factor of up to 10 was achievable in the vicinity of plasmon nanostructures. ${ }^{13-15}$ However, the origin of this effect remained obscure due to poorly defined resonance conditions; i.e., this effect could be associated with either the Purcell effect, the enhanced excitation efficiency, or both these factors. At the same time, designing a nanostructure with a specific position of the plasmon resonance relative to the excitation or PL wavelength may enable one to separate these two plasmoninduced effects. Following this strategy, many studies have dealt with the effect of accelerated radiative recombination of QD excitons near the plasmon nanostructures (the Purcell effect). However, in this regime, a significant increase in the PL intensity can occur only for QDs with an initially low $\mathrm{PL}$ quantum yield (QY). ${ }^{5} \mathrm{~A}$ representative example of this was observed for a QD-Ag film system. ${ }^{16}$ In this structure, a 50 -fold increase in the QD PL intensity was observed near the array of circular Ag nanoislands on an Ag film providing matching the QD emission and plasmon resonance. At the 
same time, initially, QDs emitted very weakly. It should further be noted that the

plasmon-induced Purcell effect may also help to overcome one of the main limitations of QDs, the presence of a low-luminescent "dark" fraction. ${ }^{17}$ Indeed, charging of QDs is a common problem, which, due to the high-rate nonradiative Auger process, leads to a decrease in the PL QY of part of the QD ensemble to near-zero values. ${ }^{18}$ This is a result of the high-rate nonradiative Auger process between excitons and excess charge carriers inside the QD core. The increase in the radiative rate due to the Purcell effect should decrease the probability of the Auger process and increase in the PL QY of these "dark" charged QDs. ${ }^{19,20}$

A completely different approach is to increase the PL intensity by enhancing the excitation efficiency near PNPs. The increase in the excitation efficiency can be as high as 10 - to $24-$ fold $^{3,21}$ however, the PL increase associated with this effect is usually only 2- to 4-fold. ${ }^{22,23}$ The reason for this is the reduction of the PL QY due to the plasmoninduced acceleration of nonradiative recombination caused by energy transfer, which is not compensated by the Purcell effect, and, hence, restrains the resulting increase in the PL intensity. Thus, both effects have strong limitations in terms of the control of QD PL: the Purcell effect cannot significantly increase the PL of QDs with an initially high 
QY, while enhanced excitation is accompanied by a decrease in the $Q Y$ of the $Q D$ ensemble. We assume that the optimal solution would be a regime where the excitation enhancement is accompanied by a simultaneous acceleration of radiative recombination, leading to the compensation for the deficiencies of both effects considered above. In this scenario the acceleration of radiative recombination will increase the PL QY even in the case of plasmon-induced quenching. At the same time, the enhancement of the excitation efficiency will considerably brighten the PL of QDs, even those with an initially high QY.

Some recent studies have attempted to investigate the combination of both effects in a colloidal solution of Au nanorods and QDs. ${ }^{24}$ However, the properties of QD PL in solutions and thin films can be very different. In another study, a 10-fold increase in the PL intensity in the system consisting of QDs and an array of Au nanorods was explained as a result of an alledged combination of all conceivable plasmon-induced effects. ${ }^{25}$ However, in this case, the plasmon spectrum was resonantly overlapped with the PL band but not with the excitation line, which implies only one interaction regime. The most representative examples of plasmon-induced PL enhancement, together with resonance conditions, are summarized in Table S1. Nevertheless, it should be noted 
that, to provide resonance matching of the plasmon spectrum with both excitation and PL in materials where only one type of PNPs is used, the excitation wavelength should be very close to the PL band. Unfortunately, in this case, one of the main advantages of QDs, the giant Stokes shift of PL from excitation, is lost, as is the possibility of exciting the PL of QDs of different sizes using only one light source with a high photon energy. Here, we fabricated hybrid thin films of QDs integrated into a poly [methyl methacrylate] (PMMA) matrix and studied how in situ deposition of PNPs with different plasmon resonance positions relative to the excitation wavelength and the QD PL band affected the PL intensity. As a result, we have shown that the enhancement of QD PL was the highest in the case where the local field enhancement effect led to a higher excitation rate of QDs and, in the same structure, the Purcell effect simultaneously prevented a strong decrease in the QY of QD PL. The resulting PL enhancement (14.2-fold) was much higher than the sum of the PL enhancement factors for the excitation enhancement (1.7-fold) and the Purcell effect (4.6-fold) separately and even higher than their product, for which we have provided rationale.

In our experiments we used QDs with the CdSe(core)/ZnS/CdS/ZnS(multishell) structure with a PL maximum at $560 \mathrm{~nm}$ (Figure 1a) and the first exciton peak at $536 \mathrm{~nm}$ 
(Figure 1b). The PL decay of QDs in a hexane solution (Figure 1c) was nearmonoexponential, with a lifetime of $30 \mathrm{~ns}$. The details of QD synthesis and preparation are presented in the Supporting Information (SI) file.
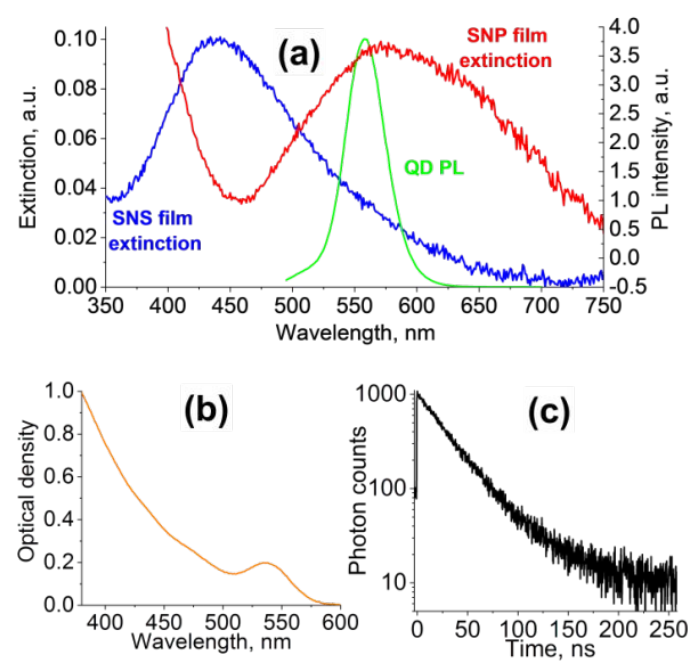

Figure 1. Optical properties of nanoparticles. (a) The extinction spectra of silver nanospheres (blue) and silver nanoplates (red) and the spectrum of QD PL (green line). (b) The extinction spectrum of QD PL. (c) The decay kinetics of QD PL. It is known that the effect of PNPs on the PL of QDs strongly depends on the distance between the QDs and PNP films. ${ }^{21,26}$ Thus, we used PMMA films of different thicknesses to check whether the observed effects actually resulted from the plasmonexciton interaction. The QD-in-PMMA films were coated with PNPs in situ during PL measurements by drop-casting of PNP solutions onto the QD-in-PMMA films. Thus, the 
time-resolved PL signals were detected from the same areas of QD-in-PMMA films

before and after their interaction with PNPs. This allowed us to avoid the possible errors

related to the inhomogeneous distribution of QDs in the film. The details of

spectroscopic measurements are presented in the SI file.

To cover QD-in-PMMA films, we used two types of PNPs with different spectral positions of the plasmon band: silver nanospheres (SNSs) and silver nanoplates (SNPs). Details of the SNS and SNP syntheses, as well as their extinction spectra in stock solutions, are presented in the SI. Three groups of samples were studied: films covered with SNSs alone (Figure 2a), films covered with SNPs alone (Figure 2b), and films covered with a mixture of SNSs and SNPs in equal proportions (Figure 2c). The photographs and optical microscopy images of PNP solutions and thin films deposited onto QD-in-PMMA substrates are presented in Figure S7. Each group comprised QD-inPMMA films with three thicknesses: 5,10 , and $20 \mathrm{~nm}$. Our sample preparation routine ensured that all QDs were distributed in the plane of the glass substrate and immobilized there by coating a thin PMMA layer with a thickness of approximately $5 \mathrm{~nm}$. QD-in-PMMA films with thicknesses of 10 and $20 \mathrm{~nm}$ were obtained by coating this QDin-PMMA film with additional PMMA spacer layers (see the SI). The details of QD-in- 
PMMA films preparation are presented in the SI file. Transmission electron microscopy (TEM) images of all PNPs are presented in Figure 2. Before the main experiments, the extinction spectra of PNPs deposited on "blank" (i.e., not containing QDs) PMMA films were measured. Figure 1a shows that the QD PL band largely overlaps only with the SNP resonance, which is prerequisite for weak light-matter coupling and the Purcell effect. On the other hand, the plasmon band of the SNS film largely overlaps with the excitation wavelength ( $485 \mathrm{~nm}$ ), which is a precondition for the plasmon enhancement of the excitation electromagnetic field near the film surface.
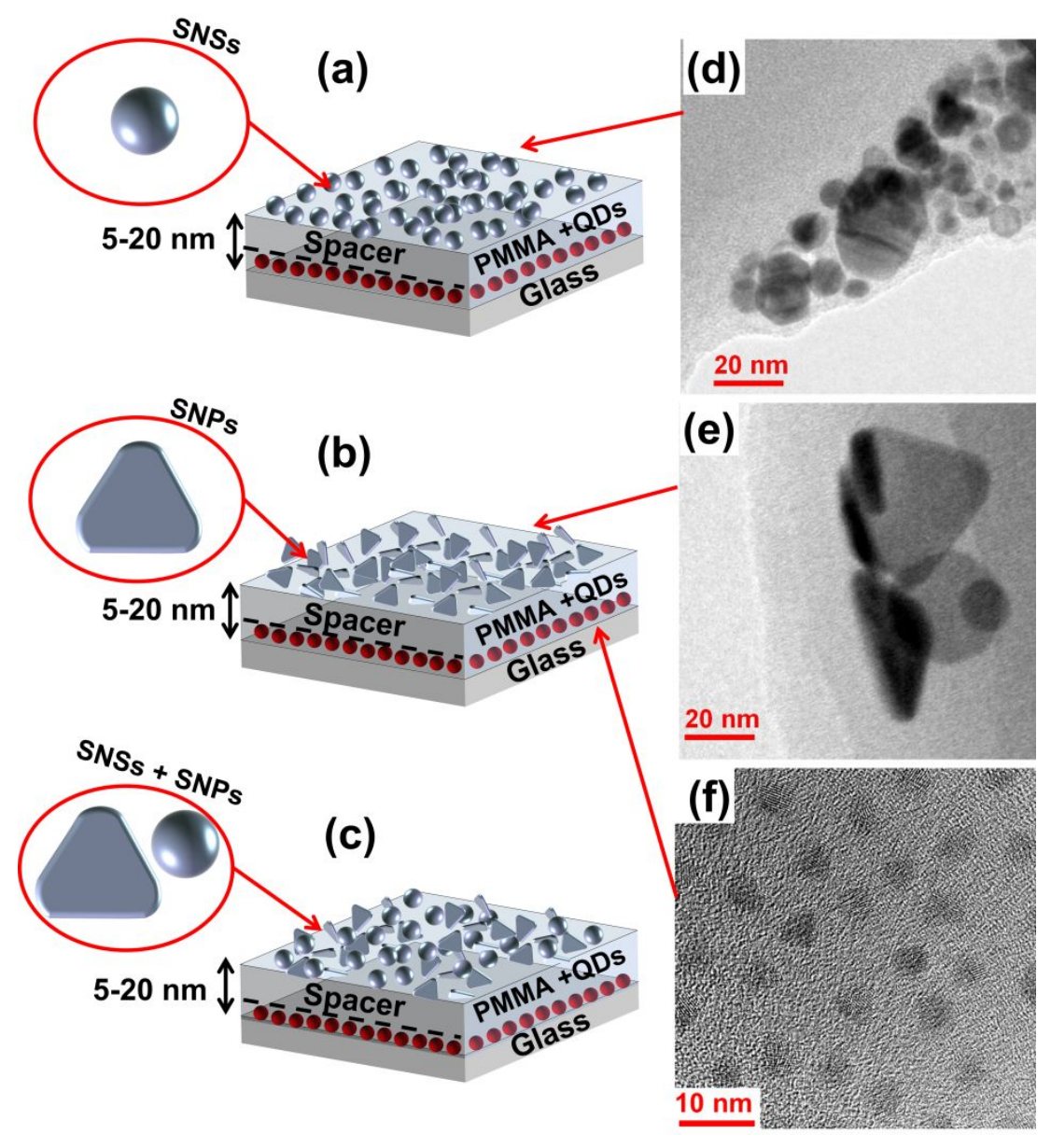
Figure 2. Schematic representation of the thin-film structures studied. Panels a, b, and c show the sketches of the samples with different PNPs: SNSs (a), SNPs (b), and a mixture of SNSs and SNPs (c). Panels $d$, e, and f show the TEM images of the nanoparticles used: SNSs, SNPs, and QDs, respectively.

Figure 3a shows the PL signal collected by scanning a $80 \times 80 \mu \mathrm{m}$ area of a QD-inPMMA film with a total thickness of $5 \mathrm{~nm}$ (black line). $30 \mu \mathrm{l}$ of the SNS solution was then drop-casted onto the QD-in-PMMA structure placed directly above the objective lens, without any changes in the position of the film or the lens. After the SNS film had dried, the PL signal was measured again from exactly the same area of the sample at the same excitation power (the red line in Figure 3a). It turned out that, in the presence of SNSs, the intensity of QD PL was higher by $150 \%$ (which translates into a 2.5 -fold enhancement). The same experiments were carried out on QD-in-PMMA films with thicknesses of $10 \mathrm{~nm}$ (Figure 3b) and $20 \mathrm{~nm}$ (Figure 3c). We should note that the amplitudes of the control PL signals (black lines in the Figure 3) slightly differ for different types of the studied samples because of the differences in the signal collection times, which were optimized for each particular film. However, these variations do not compromise our conclusions about the observed plasmon-induced effects, because the 
signal collection time for each of the studied sample was the same before and after the PNP application on top of the regions of interest. We should also point out that the plasmon-induced changes in the PL lifetimes (Table S2) were much greater than the standard deviation of PL lifetime of the control signal, i.e., the standard deviation of 0.7 ns for the mean PL lifetime of 6.6 ns. In the case of $10-n m$ films, the increase in the total PL intensity was $70 \%$ (Figure 3b); however, in the case of $20-\mathrm{nm}$ films, the total PL intensity was, conversely, decreased by $15 \%$ (Figure 3c). This finding is not surprising, because the excitation field enhancement can only be efficient at short distances between the fluorophore and PNPs and the effect of the plasmon-induced quenching may be more long-range than the field enhancement effect. ${ }^{26}$ Another effect to be taken into account is the water-induced PL quenching, which can be due to infiltration of water through the thin PMMA film (assuming that the PMMA spacer film is a filter-like structure and the surface density of pores is approximately the same for films 5,10 , and $20 \mathrm{~nm}$ in thickness). ${ }^{27}$ The combination of these effects led to enhancement of the PL intensity at the QD-in-PMMA film thicknesses of 5 and $10 \mathrm{~nm}$ but to PL quenching in the $20-\mathrm{nm}$ film. The nonradiative relaxation also manifested itself in a shortening of the PL lifetime (Table S2 in SI). These results also mean that the observed PL brightness increase was 
much lower than expected due to the excitation enhancement and could be much stronger at a higher radiative rate.

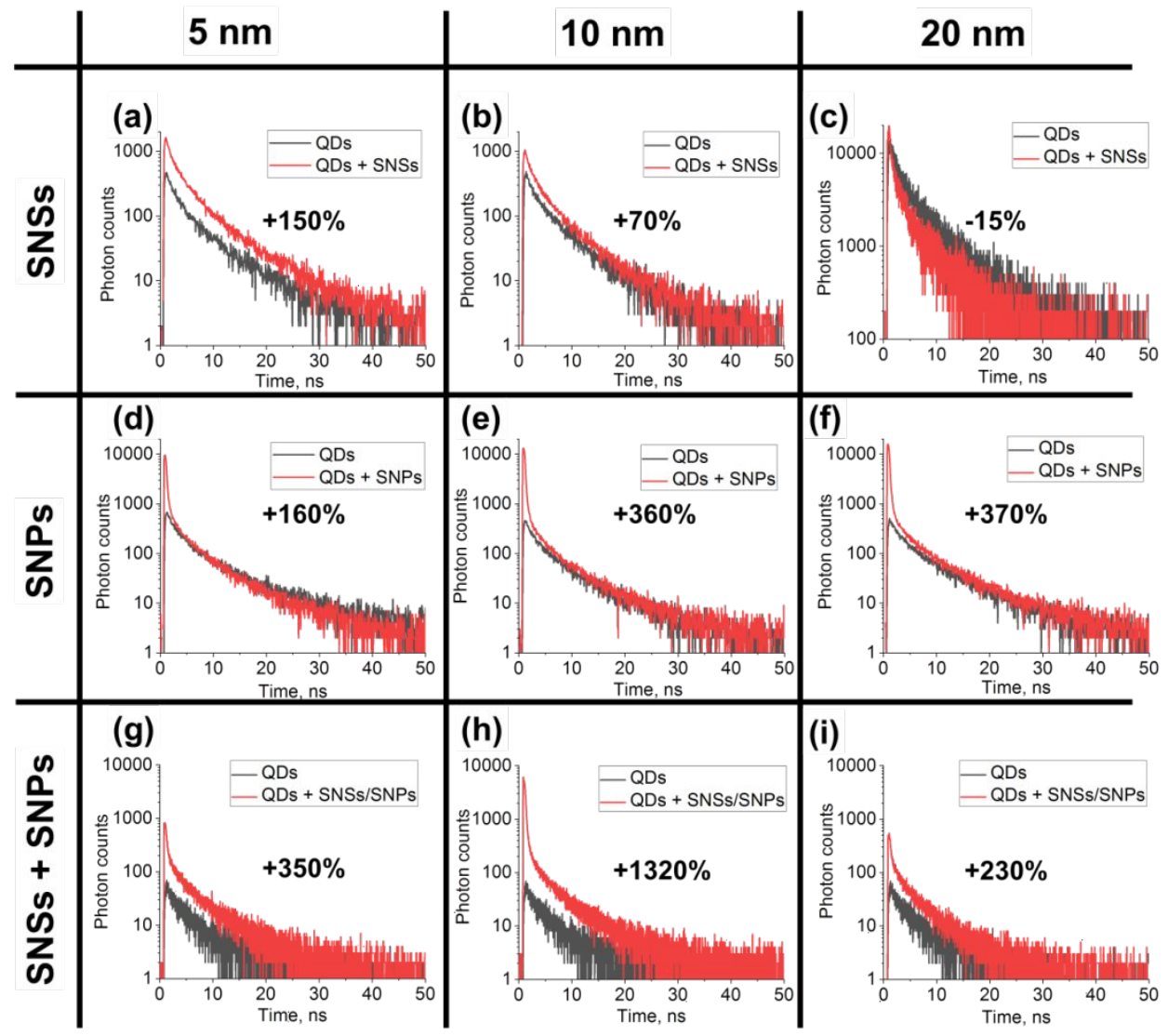

Figure 3. Time-resolved PL signals from QD-in-PMMA films before (black lines) and after (red lines) deposition of PNPs for different PNP types and different QD-in-PMMA film thicknesses. (a-c) PL signals from samples with deposited SNSs and different thicknesses: (a) $5 \mathrm{~nm}$, (b) $10 \mathrm{~nm}$, and (c) $20 \mathrm{~nm}$. (d-f) PL signals collected from samples with deposited SNPs and different thicknesses: (d) $5 \mathrm{~nm}$, (e) $10 \mathrm{~nm}$, and (f) 20 nm. (g-i) PL signals collected from samples with a deposited SNS-SNP mixture and different thicknesses: (g) $5 \mathrm{~nm}$, (h) $10 \mathrm{~nm}$, and (i) $20 \mathrm{~nm}$. 
The experiments with SNP-containing samples were carried out following the same procedure; i.e., the PL signals from QD-in-PMMA films of different thicknesses were measured from the same areas (Figures $3 d-3 f$ ) before (black lines) and after (red lines) SNP deposition. Figures $3 d-3 f$ clearly show that, at all film thicknesses, the addition of SNPs led to a drastic alteration of the PL decay, which can be seen as the appearance of an ultrafast component with a high signal amplitude and a response time close to the instrument response function (IRF) of the detection system (Table S2). At the same time, the long-time components of the PL decay were shorter for thinner QD-in-PMMA films, which implies the Purcell effect, but their values are still longer than the IRF. This can be explained if we take into account that QD ensembles often contain two types of QDs: bright QDs with a relatively high PL QY and a "dark fraction" of QDs that are in the low- or non-luminescent state. The latter are charged permanently or at least for long periods of time and, consequently, have a high-rate Auger-like nonradiative exciton recombination channel. ${ }^{18,28}$ We were unable to observe the $\mathrm{PL}$ response of the QDs from the "dark fraction" before interaction with SNPs due to their initially very low PL QY. However, for QD samples with a lower initial QY (the "dark fraction"), the increase in the radiative recombination rate due to the Purcell effect could cause a substantial 
growth of the PL QY, and, in this case, the total enhancement of QY would be higher than that observed in the case of SNS-covered samples. Thus, a strong Purcell effect could lead to a considerable increase in the contribution of the brightened "dark" part of QDs to the overall PL signal. Along with this, such an increased contribution of the originally "dark" fraction of QDs with an initially very short PL lifetime could lead to the appearance of an ultrafast component in the PL signal.

To confirm our assumption, we acquired a series of PL images of selected areas of interest before and after SNP deposition (Figure 4). It can be seen that the addition of SNSs caused a distinct though moderate increase in the brightness of the QD film (Figures $4 a$ and $4 b$ ). In contrast, the deposition of SNPs led to a substantial growth of the bright fraction of the image (Figures $4 c$ and $4 d$ ). This brightening is consistent with the assumed increased contribution of initially "dark" QDs, which were previously undetectable. We believe that the bright and non-uniform patterns observed, which are shown in Figures 4b, 4d, and 4f, most likely reflect the distribution of the PNP aggregates on the surface of QD-in-PMMA films. 


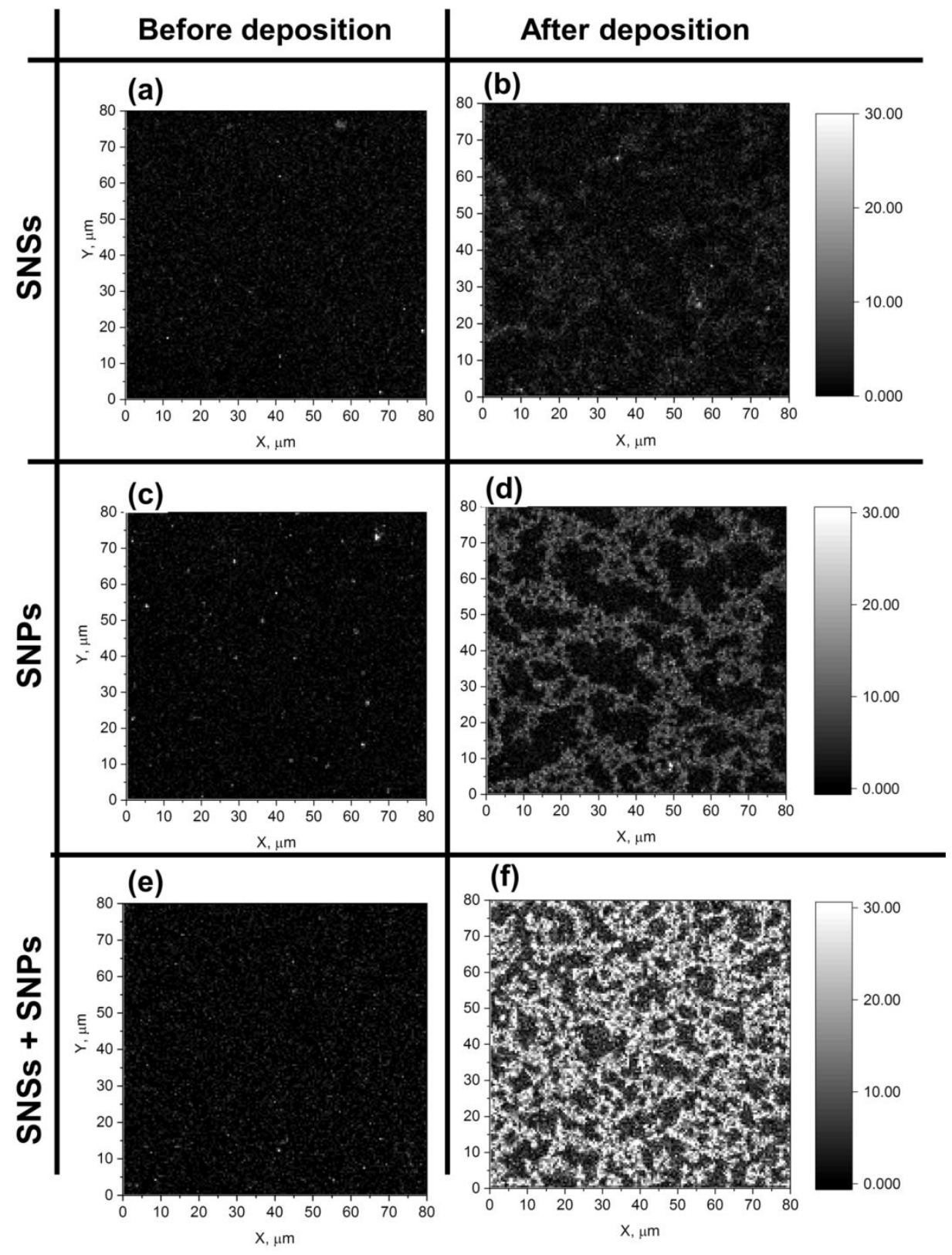

Figure 4. Confocal microscopy PL images of QD-in-PMMA films with a total thickness of $10 \mathrm{~nm}$ before $(\mathrm{a}, \mathrm{c}, \mathrm{e})$ and after $(\mathrm{b}, \mathrm{d}, \mathrm{f})$ deposition of PNPs: $(\mathrm{a}, \mathrm{b})$ before and after SNS deposition, (c, d) before and after SNP deposition, (e, f) before and after deposition of the mixture of SNSs and SNPs. 


\begin{abstract}
Despite the random distribution of SNPs on the surface of the QD-in-PMMA film, the interaction of any individual QD with neighboring SNPs is characterized by a high
\end{abstract} Purcell factor even at plasmon-exciton distances as long as $135 \mathrm{~nm} \cdot{ }^{20}$ Therefore, the observed PL enhancement is the averaged cumulative result of all individual QD-SNP interactions. It is also worth noting that the increase in the PL intensity of the SNPcovered samples of the QD-in-PMMA structures with thicknesses of 10 and $20 \mathrm{~nm}$ $(+360 \%$ and $+370 \%$, respectively) were higher than that observed in the $5-\mathrm{nm}$ sample. We believe that this is a result of the distance-dependent "tug of war" between plasmoninduced energy transfer, which increases the nonradiative recombination rate, and the Purcell effect, which, in turn, raises the radiative recombination rate..$^{20,26,29,30}$ However, in accordance with the theory of plasmon-exciton interaction, for PNPs with morphologies and sizes other than those studied here, the optimal thickness of the spacer will be different. In addition, this parameter could potentially be affected by the density of PNPs on the surface of the QD-in-PMMA film.

The maximum QD PL enhancement (370\%), which was observed when the SNPs were used, was much higher than that in the case of the SNSs (150\%). The reason for this difference is the SNP capacity for increasing the QY of "dark" QDs. In both cases, 
metal-induced PL quenching (an increase in the nonradiative relaxation rate) occurred.

In the case of the SNSs, this leads to a drop of the $\mathrm{QY}$, because the radiative relaxation rate in the presence of the nanospheres was unchanged. Thus, the resultant PL enhancement was the product of the reduced QY and the increased excitation intensity, which limits the PL enhancement for both initially highly luminescent "bright" QDs and non-luminescent "dark" QDs that are present in the sample. As for the SNPs, the roles of the former two factors are additionally increased. Indeed, the increase in the radiative relaxation rate due to the Purcell effect can certainly overwhelm the metal-induced decrease in the PL QY of "bright" QDs and, additionally, switch on the initially nonluminescent "dark" QDs to make them "bright”.

However, the most outstanding results were obtained in experiments carried out before and after deposition of the mixture made of equal amounts of SNSs and SNPs onto the QD-in-PMMA films. The extinction spectrum of the mixed SNS/SNP film is shown in Figure S6 (black solid line). To demonstrate that this spectrum is the result of superposition of two independent plasmon resonances originating from the SNSs and SNPs, we have fitted the experimental spectrum by two Gaussian bands (dashed red and green lines in Figure S6). The sum of these two Gaussian bands (dashed blue line 
in Figure S6) is in good agreement with the experimental spectrum. The positions of these two bands are in good agreement with the experimental extinction spectra of both SNS and SNP films (Figure 1a), which proves that the mixed SNS/SNP film exhibits resonances with both $\mathrm{PL}$ excitation and the PL emission of the QDs. Time-resolved measurements revealed that, after deposition of the SNS/SNP mixture, PL signals contain combined features caused by the SNS and SNP effects (Figures 3g-3i). First, the high-amplitude ultrafast component of PL decay was observed, as in the case of the effect of deposition of SNPs alone. Moreover, the amplitude of the long-lifetime component of the PL decay increased much more than in the cases when only SNSs or SNPs were deposited (Table S2). This feature can be explained by the fact that, in these samples, the Purcell effect compensates for the plasmon-induced PL quenching and the quenching does not interfere with the excitation enhancement. The PL image of a sample covered with SNS/SNP blend (Figure $4 f$ ) is similar to the picture of the SNP sample (Figure 4d), where the "bright" fraction of the image was larger than in the SNScovered film (Figure 4b). However, the overall brightness of the structure with the PNP blend was significantly higher than in the cases of SNS- and SNP-covered samples. This result implies that the effect caused by the SNS/SNP mixture leads not only to an 
increased contribution of the brightened the "dark fraction" of the QD ensemble caused by the Purcell effect, but also to an additional increase in the PL intensity due to the excitation enhancement.

The most outstanding result was obtained for the sample with a QD-in-PMMA film 10 $\mathrm{nm}$ in thickness, in which the PL enhancement was as much as $+1320 \%$.

Extraordinarily, this value is much higher than the simple sum of the enhancements observed for this thickness in films covered with only SNSs $(+70 \%)$ or SNPs $(+360 \%)$.

This finding can be explained by the simultaneous increase in the excitation and radiative recombination rates in the QD-in-PMMA film with an SNS/SNP layer. Indeed, in the case of PL increase in the samples with only SNSs as a surface layer, the excitation intensity $I_{e x}$ can be increased due to the well-known effect of plasmoninduced amplification of the intensity of the electromagnetic field near the SNS surface by the factor $K$, and the PL enhancement factor $F_{E E}$ can be expressed by the following equation (for derivation of this and following equations, see SI):

$F_{E E} \approx \kappa \cdot \frac{k_{r a d}+k_{n r}}{k_{r a d}+k_{n r}+k_{E T}}$

where $k_{\text {rad }}$ is the radiative recombination rate, $k_{n r}$ is the rate of internal nonradiative relaxation due to the inner or surface defects, and $k_{E T}$ is the metal-induced nonradiative 
energy transfer rate in the proximity of PNPs. Due to the Purcell effect, in the samples covered with SNPs alone, the $k_{\text {rad }}$ should be multiplied by the Purcell factor $F$. Thus, the observed increase in the number of collected photons as a result of the rise of the QY and the PL enhancement factor $F_{P E}$ is

$F_{P E} \approx \frac{F \cdot k_{r a d}+F \cdot k_{n r}}{F \cdot k_{r a d}+k_{n r}+k_{E T}}$

In the most interesting case, when the SNS/SNP mixture is used to cover the QD-inPMMA films, both the excitation field amplitude will be increased and the Purcell effect will take place. In this case the amplification coefficient can be expressed as

$F_{\text {sum }} \approx \kappa \cdot \frac{F \cdot k_{\text {rad }}+F \cdot k_{n r}}{F \cdot k_{\text {rad }}+k_{n r}+k_{E T}}$.

From eq 3 it is evident that, for the QD-in-PMMA films covered with the SNS/SNP mixture, the resulting PL enhancement is higher than mere superposition of the abovementioned individual enhancements. Moreover, the PL amplification factor (eq 3) is higher than the result of simple multiplication of the PL enhancements observed in the cases of excitation enhancement (eq 1) and Purcell effect (eq 2) separately. Thus, the effect the excitation enhancement alone on the PL intensity is limited by lowering of the PL QY, but a substantial increase in the radiative recombination rate not only restrains 
the decrease of the QY, but can also lead to its growth. As a result, the total "synergistic" impact of the superposition of both effects is much stronger than the influence of the excitation enhancement or the Purcell effect separately.

In conclusion, we have studied the "synergistic" effect of the plasmon-induced enhancements of excitation and radiative recombination rates on the QD PL brightness. For this purpose, we fabricated thin PMMA films with incorporated QDs and PNPs deposited onto polymer spacers of various thicknesses. For each film thickness, the PL signals from exactly the same areas of these films were studied before and after initiation of QD-PNP interaction. To investigate the effect of spectral overlap, we used two types of deposited PNPs: SNSs, whose plasmon resonance overlapped with the excitation wavelength, and SNPs, with the plasmon band overlapping with the QD PL line. We found that, for samples in which only SNSs covered the QD-in-PMMA films, the plasmon-induced PL increase was up to $150 \%$ and was weakening with increasing thickness of the QD-in-PMMA films. In this case, the observed growth of the PL intensity is the result of the excitation enhancement and is restricted by the nonradiative recombination due to the plasmon-induced energy transfer. The effect of SNPs on the PL signal of QDs was more complex. In this case, due to the Purcell effect, a significant 
fraction of initially non-luminescent QDs began to contribute to the overall PL signal with an ultrashort lifetime. This led to an expansion of the bright area in PL images and an enhancement of PL of up to $370 \%$. Moreover, in this case, the maximum PL intensification was achieved for thicker QD-in-PMMA films than in the case of the SNScovered samples. The main and most remarkable result of our study was observed for a composite film where a mixture of SNSs and SNPs served as a cover layer for the QDin-PMMA structure. In these samples, we observed both an increase in the number of luminescent QDs and an increase in the PL intensity of the initially "bright" QDs. Moreover, the maximum PL increase observed in the samples covered with a blend of SNSs and SNPs was $1320 \%$, which is much higher than the result of a simple superposition of the PL enhancements induced by SNS (70\%) and SNP (360\%) alone. We explained this result by a simultaneous increase in the QD absorption due to the enhanced excitation and increase in the QY due to the Purcell effect. Thus, the metalinduced quenching, which decreases the PL QY in the case of the use of SNSs alone, was compensated for by the Purcell effect. Moreover, the QDs with an initially high QY were brightened by an excitation enhancement stronger than in the case where only SNPs were used. Finally, we have provide rationale that the synergy of the excitation 
enhancement and the Purcell effect leads to a total PL enhancement that is even higher than the result of multiplication of the PL enhancement factors in the cases of using SNSs and SNPs alone. The demonstrated synergy of the excitation enhancement and the Purcell effect as a tool for controlling the efficiency of spontaneous emission of thin hybrid QD-polymer films can be used for numerous applications in the fields of biosensing, optoelectronics, and quantum information. Moreover, optimal combinations of plasmon nanostructures with different plasmon resonances will allow flexible adaptation of the designed systems for the required spectral conditions of excitation and the resulting $\mathrm{PL}$.

\section{ASSOCIATED CONTENT}

Supporting Information. A brief review of the examples of plasmon-induced PL enhancement; details of nanocrystal sample synthesis; details of spectroscopic measurements; details of QD-in-PMMA film preparation; details of the AFM profiling measurements of the thicknesses of thin films; details of the biexponential fitting of the PL decay kinetics; analytical explanation of the plasmon-induced enhancement synergy. (PDF) 


\title{
AUTHOR INFORMATION
}

\section{Corresponding Authors}

Victor Krivenkov: vkrivenkov@list.ru

Yury Rakovich: yury.rakovich@ehu.eus

\section{ORCID}

\author{
Victor Krivenkov: 0000-0003-0280-2296 \\ Pavel Samokhvalov: 0000-0002-2878-8376 \\ Yury Rakovich: 0000-0003-0111-2920 \\ Igor Nabiev: 0000-0002-8391-040X
}

Notes

The authors declare no competing financial interests.

\section{ACKNOWLEDGMENTS}

The authors thank D. Dyagileva for the help with preparation of the QD-in-PMMA thin films, and I.S. Vaskan for assistance in AFM profiling measurements. The financial support from the Ministry of Science and Higher Education of the Russian Federation through grant no. 14.Y26.31.0011 is acknowledged. Y.R. acknowledges the support from the Basque Government (grant no. IT1164-19). I.N. is grateful to the Université de 
Reims Champagne-Ardenne, the Ministry of Higher Education, Research and Innovation, and the Conseil Regional de Grand Est of France for support. We thank Vladimir Ushakov for the help with technical preparation of the manuscript.

\section{REFERENCES}

(1) Maier, S. A. Plasmonics: Fundamentals and Applications; Springer: New York, 2007.

(2) Gaponenko, S. V.; Guzatov, D. V. Colloidal Plasmonics for Active Nanophotonics. Proc. IEEE 2020, 108 (5), 704-720.

(3) Chen, Y.; Munechika, K.; Plante, J.-L.; Munro, A. M.; Skrabalak, S. E.; Xia, Y.; Ginger, D. S. Excitation Enhancement of CdSe Quantum Dots by Single Metal Nanoparticles. Appl. Phys. Lett. 2008, 93 (5), 053106.

(4) Chen, Y.; Munechika, K.; Ginger, D. S. Dependence of Fluorescence Intensity on the Spectral Overlap between Fluorophores and Plasmon Resonant Single Silver Nanoparticles. Nano Lett. 2007, 7(3), 690-696.

(5) Munechika, K.; Chen, Y.; Tillack, A. F.; Kulkarni, A. P.; Plante, J.-L.; Munro, A. M.; Ginger, D. S. Spectral Control of Plasmonic Emission Enhancement from Quantum Dots near Single Silver Nanoprisms. Nano Lett. 2010, 10(7), 25982603.

(6) Dovzhenko, D.; Martynov, I.; Samokhvalov, P.; Osipov, E.; Lednev, M.; 
Chistyakov, A.; Karaulov, A; Nabiev, I. Enhancement of Spontaneous Emission of Semiconductor Quantum Dots inside One-Dimensional Porous Silicon Photonic Crystals. Opt. Express 2020, 28 (15), 22705-22717.

(7) Bozhevolnyi, S. I.; Khurgin, J. B. Fundamental Limitations in Spontaneous Emission Rate of Single-Photon Sources. Optica 2016, 3(12), 1418.

(8) Yang, Z.; Gao, M.; Wu, W.; Yang, X.; Sun, X. W.; Zhang, J.; Wang, H. C.; Liu, R. S.; Han, C. Y.; Yang, H.; Li, W. Recent Advances in Quantum Dot-Based LightEmitting Devices: Challenges and Possible Solutions. Mater. Today 2019, 24, 6993.

(9) Kim, N. Y.; Hong, S. H.; Kang, J. W.; Myoung, N.; Yim, S. Y.; Jung, S.; Lee, K.; Tu, C. W.; Park, S. J. Localized Surface Plasmon-Enhanced Green Quantum Dot Light-Emitting Diodes Using Gold Nanoparticles. RSC Adv. 2015, 5(25), 1962419629.

(10) Yang, X.; Hernandez-Martinez, P. L.; Dang, C.; Mutlugun, E.; Zhang, K.; Demir, H. V.; Sun, X. W. Electroluminescence Efficiency Enhancement in Quantum Dot Light-Emitting Diodes by Embedding a Silver Nanoisland Layer. Adv. Opt. Mater. 2015, $3(10), 1439-1445$.

(11) Guan, W.; Zhou, W.; Lu, J.; Lu, C. Luminescent Films for Chemo- and Biosensing. Chemical Society Reviews 2015, 44 (19), 6981-7001.

(12) Dey, S.; Zhao, J. Plasmonic Effect on Exciton and Multiexciton Emission of Single Quantum Dots. J. Phys. Chem. Lett. 2016, 7(15), 2921-2929. 
(13) Kulakovich, O.; Strekal, N.; Yaroshevich, A.; Maskevich, S.; Gaponenko, S.;

Nabiev, I.; Woggon, U.; Artemyev, M. Enhanced Luminescence of CdSe Quantum

Dots on Gold Colloids. Nano Lett. 2002, 2(12), 1449-1452.

(14) Biteen, J. S.; Pacifici, D.; Lewis, N. S.; Atwater, H. A. Enhanced Radiative

Emission Rate and Quantum Efficiency in Coupled Silicon Nanocrystal-

Nanostructured Gold Emitters. Nano Lett. 2005, 5(9), 1768-1773.

(15) Ito, Y.; Matsuda, K.; Kanemitsu, Y. Mechanism of Photoluminescence

Enhancement in Single Semiconductor Nanocrystals on Metal Surfaces. Phys. Rev. B 2007, 75(3), 1-4.

(16) Song, J. H.; Atay, T.; Shi, S.; Urabe, H.; Nurmikko, A. V. Large Enhancement of Fluorescence Efficiency from CdSe/ZnS Quantum Dots Induced by Resonant Coupling to Spatially Controlled Surface Plasmons. Nano lett 2005, 5(8), 15571561.

(17) Yao, J.; Larson, D. R.; Vishwasrao, H. D.; Zipfel, W. R.; Webb, W. W. Blinking and Nonradiant Dark Fraction of Water-Soluble Quantum Dots in Aqueous Solution. Proc. Natl. Acad. Sci. 2005, 102 (40), 14284-14289.

(18) Krivenkov, V.; Samokhvalov, P.; Zvaigzne, M.; Martynov, I.; Chistyakov, A.; Nabiev, I. Ligand-Mediated Photobrightening and Photodarkening of CdSe/ZnS Quantum Dot Ensembles. J. Phys. Chem. C 2018, 122 (27), 15761-15771.

(19) Ma, X.; Tan, H.; Kipp, T.; Mews, A. Fluorescence Enhancement, Blinking Suppression, and Gray States of Individual Semiconductor Nanocrystals Close to 
Gold Nanoparticles. Nano Lett. 2010, $10(10)$, 4166-4174.

(20) Krivenkov, V.; Dyagileva, D.; Samokhvalov, P.; Nabiev, I.; Rakovich, Y. Effect of Spectral Overlap and Separation Distance on Exciton and Biexciton Quantum Yields and Radiative and Nonradiative Recombination Rates in Quantum Dots Near Plasmon Nanoparticles. Ann. Phys. (Berlin) 2020, 532 (8), 2000236.

(21) Jin, S.; DeMarco, E.; Pellin, M. J.; Farha, O. K.; Wiederrecht, G. P.; Hupp, J. T. Distance-Engineered Plasmon-Enhanced Light Harvesting in CdSe Quantum Dots. J. Phys. Chem. Lett. 2013, 4 (20), 3527-3533.

(22) Sun, D.; Tian, Y.; Zhang, Y.; Xu, Z.; Sfeir, M. Y.; Cotlet, M.; Gang, O. LightHarvesting Nanoparticle Core-Shell Clusters with Controllable Optical Output. ACS Nano 2015, $9(6), 5657-5665$.

(23) Liang, H. Y.; Zhao, H. G.; Li, Z. P.; Harnagea, C.; Ma, D. L. Silver Nanoparticle Film Induced Photoluminescence Enhancement of Near-Infrared Emitting PbS and PbS/CdS Core/Shell Quantum Dots: Observation of Different Enhancement Mechanisms. Nanoscale 2016, $8(9)$, 4882-4887.

(24) Trotsiuk, L.; Muravitskaya, A.; Kulakovich, O.; Guzatov, D.; Ramanenka, A.; Kelestemur, Y.; Demir, H. V; Gaponenko, S. Plasmon-Enhanced Fluorescence in Gold Nanorod-Quantum Dot Coupled Systems. Nanotechnology 2020, 31 (10), 105201.

(25) Peng, B.; Li, Z.; Mutlugun, E.; Hernández Martínez, P. L.; Li, D.; Zhang, Q.; Gao, Y.; Demir, H. V.; Xiong, Q. Quantum Dots on Vertically Aligned Gold Nanorod 
Monolayer: Plasmon Enhanced Fluorescence. Nanoscale 2014, 6(11), 55925598.

(26) Guzatov, D. V; Vaschenko, S. V; Stankevich, V. V; Lunevich, A. Y.; Glukhov, Y. F.; Gaponenko, S. V. Plasmonic Enhancement of Molecular Fluorescence near Silver Nanoparticles: Theory, Modeling, and Experiment. J. Phys. Chem. C2012, $116(19)$, 10723-10733.

(27) Pechstedt, K.; Whittle, T.; Baumberg, J.; Melvin, T. Photoluminescence of Colloidal CdSe/ZnS Quantum Dots: The Critical Effect of Water Molecules. J. Phys. Chem. C 2010, 114 (28), 12069-12077.

(28) Murthy, A. V. R.; Patil, P.; Datta, S.; Patil, S. Photoinduced Dark Fraction Due to Blinking and Photodarkening Probability in Aqueous CdTe Quantum Dots. J. Phys. Chem. C 2013, $117(25), 13268-13275$.

(29) Krivenkov, V.; Goncharov, S.; Samokhvalov, P.; Sánchez-Iglesias, A.; Grzelczak, M.; Nabiev, I.; Rakovich, Y. Enhancement of Biexciton Emission Due to LongRange Interaction of Single Quantum Dots and Gold Nanorods in a Thin-Film Hybrid Nanostructure. J. Phys. Chem. Lett. 2019, 10, 481-486.

(30) Song, M.; Wu, B.; Chen, G.; Liu, Y.; Ci, X.; Wu, E.; Zeng, H. Photoluminescence Plasmonic Enhancement of Single Quantum Dots Coupled to Gold Microplates. J. Phys. Chem. C 2014, 118 (16), 8514-8520. 